\title{
Balón intragástrico ajustable como tratamiento de reducción de peso. Experiencia en un centro Mexicano
}

\author{
Noé Israel Cano-Zepeda*, Fernando Pérez-Aguilar, José Gutiérrez-Sotres, Miguel Ángel Torres-Mendoza, \\ Christian Arnoldo Carballido-Barrita, Edgar Meza-Félix, Pamela Navarro-Hernández y \\ Luis Martín Manrique-Velasco
}

Unidad de Endoscopia Gastrointestinal, Hospital Dr. Fernando Quiroz Gutiérrez, Instituto de Seguridad y Servicios Sociales de los Trabajadores del Estado, Ciudad de México, México

\begin{abstract}
Resumen
Introducción: La obesidad afecta a millones de personas y existen múltiples tratamientos para reducción de peso. Los tratamientos quirúrgicos han mostrado superioridad sobre otros. Sin embargo, el número de pacientes candidatos a cirugía es escaso. Objetivos: Evaluar la reducción de peso, índice de masa corporal (IMC), exceso de peso corporal y porcentaje de exceso de peso de los pacientes con el uso de balón intragástrico. Materiales y métodos: Se evaluaron pacientes a quienes se colocó balón intragástrico ajustable como tratamiento para reducción de peso, en un centro endoscópico mexicano durante el periodo comprendido del 1 de marzo de 2017 al 1 de marzo de 2018. Se evaluó la reducción de peso al mes 9 y 12. Se aplicó análisis estadístico con t de Student para validar la diferencia de medias inicial y final para peso, IMC y exceso de peso corporal; además se contrastaron los porcentajes de exceso de peso perdidos con las recomendaciones internacionales. En total 11 pacientes cumplieron con los criterios de inclusión y se clasificaron en dos grupos de registro a los 9 y 12 meses. Resultados: Se demostró una reducción de peso, IMC y exceso de peso corporal estadísticamente significativa a los 12 meses ( $p$ < 0.05). El porcentaje de exceso de peso perdido alcanzado a los 9 meses fue del $34.50 \%$ y a los 12 meses del 40.52\%. Conclusiones: El uso del balón intragástrico ajustable como tratamiento para reducción de peso en la población mexicana ha mostrado resultados similares a los reportados en la literatura, sin embargo, solo es una parte del manejo multidisciplinario para el manejo de la obesidad.
\end{abstract}

Palabras clave: Obesidad. Endoscopia. Balón intragástrico. Bariatría. México.

\section{Adjustable intragastric balloon like treatment for loss weight. Experience in a Mexican center}

\section{Abstract}

Introduction: Obesity affects millions of persons and there are many treatments for weight loss. The surgical treatments have shown superiority over others. Nevertheless, the number of patients who are candidates is very reduced. Objectives: Evaluate the weight loss, body mass index (BMI) and the excess body weight, such as the percentage of excess body weight loss in the patients with adjustable intragastric balloon. Materials and methods: We evaluated the patients with adjustable

\section{Correspondencia:}

*Noé Israel Cano-Zepeda

E-mail: ncanoz@ hotmail.com
Fecha de recepción: 31-01-2019

Fecha de aceptación: 02-04-2019

DOI: 10.24875/END.19000006
Disponible en internet: 06-09-2019

Endoscopia. 2019;31:102-107

www.endoscopia-ameg.com

0188-9893/@ 2019. Asociación Mexicana de Endoscopia Gastrointestinal, publicado por Permanyer México SA de CV, todos los derechos reservados. 
intragastric balloon for weight loss in an endoscopic Mexican center, from March 1, 2017 to March 1, 2018. Weight reduction was evaluated at months 9 and 12. Statistical analysis was applied with Student's $t$ to validate the difference of initial and final means for weight, BMI and excess body weight. In addition, percentage of excess body weight loss were compared with international recommendations. A total of 11 patients met the inclusion criteria and were classified into two registration groups at 9 and 12 months. Results: Weight, BMI and excess body weight reduction were statistically significant at 12 months $(p<0.05)$. The percentage of excess body weight loss reached at 9 months was $34.50 \%$ and at 12 months $40.52 \%$. Conclusions: The use of adjustable intragastric balloon as a treatment for weight loss in the Mexican population is similar to those reported in the literature, however, it is only a part of the multidisciplinary management for the treatment of obesity.

Key words: Obesity. Endoscopy. Intragastric balloon. Bariatric. México.

\section{Introducción}

La obesidad afecta mundialmente a aproximadamente 600 millones de personas ${ }^{1}$. La obesidad lleva consigo un aumento en la presencia de comorbilidades tales como diabetes mellitus, dislipidemias, hipertensión, enfermedad coronaria, síndrome de hipopnea/apnea obstructiva del sueño, osteoartrosis, esteatosis hepática no alcohólica y enfermedad por reflujo gastroesofági$\mathrm{co}^{2,3}$. En nuestro país aproximadamente el $33 \%$ de los adultos presentan obesidad (índice de masa corporal [IMC] mayor a $\left.30 \mathrm{~kg} / \mathrm{m}^{2}\right)^{4}$. Las consecuencias económicas de los problemas médicos atribuidos a la obesidad son tan importantes que en EE.UU. representa el $21 \%$ del gasto médico ${ }^{3,5}$.

En nuestro país el impacto económico de la obesidad estimado por la Secretaría de Salud en el año 2008 fue de 42 millones de pesos, directamente relacionados con comorbilidades ocasionadas por la obesidad, esto representó el $13 \%$ del costo total de gasto médico; y 25 millones de pesos indirectamente relacionados con la obesidad, secundarios a la perdida de la productividad de las personas con obesidad. También se realizó en ese momento una proyección para el año 2017, arrojando un posible gasto de 78 millones de pesos directamente relacionados con la obesidad y 73 millones de pesos indirectamente relacionados ${ }^{6,7}$.

Los objetivos de la terapia de reducción de peso son prevenir, disminuir o revertir las complicaciones asociadas con la obesidad y mejorar la calidad de vida ${ }^{8}$.

Actualmente las distintas opciones de tratamiento disponibles incluyen: cambios en el estilo de vida, farmacoterapia, tratamiento endoscópico y quirúrgico ${ }^{2,9}$. Los parámetros utilizados para medir la efectividad del tratamiento para reducción de peso son el porcentaje de exceso de peso perdido (EPP) y el porcentaje de peso corporal total perdido (PCTP) ${ }^{3}$.

Los cambios en el régimen dietético, plan de ejercicio y cambios conductuales son considerados la piedra angular de cualquier tratamiento para pérdida de peso ${ }^{3}$.
En la actualidad se ha comprobado la efectividad de los tratamientos con base en el porcentaje de PCTP y de EPP. Los cambios en el estilo de vida tienen una efectividad del $5-10 \%$ PCTP; cambios en el estilo de vida más farmacoterapia, del 4-11\% PCTP y la cirugía bariátrica, del 30-74\% EPP ${ }^{10}$.

Existen múltiples estudios que demuestran la superioridad de los tratamientos quirúrgicos sobre los demás para pérdida de peso ${ }^{3}$.

Sin embargo, solo el $1 \%$ de los pacientes obesos candidatos a la cirugía bariátrica se realizan el procedimiento ${ }^{11}$. Se han propuesto múltiples razones, costos elevados, preferencia de los pacientes y acceso al sistema de salud ${ }^{2,12}$.

En los últimos años se han popularizado alternativas endoscópicas no quirúrgicas para el tratamiento de pérdida de peso para pacientes con obesidad moderada a severa, como complemento o cuando no son suficientes los cambios en el estilo de vida ${ }^{13}$. Una de estas opciones es el balón intragástrico. Desde hace cerca de 30 años se ha utilizado con éxito como tratamiento de reducción de peso ${ }^{13,14}$.

El balón intragástrico se ha rediseñado continuamente para aumentar la pérdida de peso y mejorar la tolerancia del paciente, además de disminuir las complicaciones. El ideal para tratamiento de pérdida de peso debería de cumplir con ciertas características, tales como: a) ser de un material suave y duradero con un potencial ulcerogénico bajo, b) poseer marca radiopaca para el seguimiento y en caso de migración poder identificarlo por imagen y c) posibilidad de ajustar su tamaño ${ }^{13}$. Actualmente existen diversas opciones de balón intragástrico, cada uno con características particulares en su colocación, duración, capacidad, material interno y dispositivo de retirada.

Uno de los más comercializados y difundidos es el balón intragástrico Orbera ${ }^{\circledR}$ (Apollo endosurgery, Texas), su nombre previo fue Bioenterics ${ }^{\circledR}$ (Allergan, CA). Con capacidad de 450-700 ml de solución salina, su colocación y retirada es por vía 
endoscópica. Cuenta con una duración de 6 meses, el PCTP se encuentra entre el 10 y el $13.2 \%$. Se ha reportado la presencia de náuseas y vómitos en el $30 \%$ de los pacientes, y la necesidad de extracción temprana en un $7.5 \%$ por pobre tolerancia. La migración del balón se ha reportado en el $1.4 \%$ y perforación gástrica en el $0.1 \%$ 1,13,15-17. El punto débil que se le ha observado es la duración y en ocasiones la necesidad de recolocación para obtener una mayor pérdida de peso ${ }^{16}$.

El balón intragástrico ajustable (Spatz $3^{\circledR}$, Medical Great Neck, NY) tiene una capacidad de 400 a $700 \mathrm{ml}$ y puede permanecer intragástrico hasta 12 meses, además de tener la posibilidad de ajustar su capacidad de acuerdo con los síntomas que presente el paciente y la pérdida de peso. El porcentaje de EPP a 12 meses se ha reportado desde 45.8 a $56.7 \% \%^{14,16,18}$. Del 30 al $50 \%$ de los pacientes presentan náuseas y vómitos y del 4 al $7 \%$ no lo toleran, por lo que se retira de manera temprana ${ }^{14,16,18}$. Las complicaciones 0 incidentes relevantes (sangrado, perforación gástrica, oclusión intestinal, rotura del balón) se han reportado desde un $4.1 \%$ hasta un $15.8 \%{ }^{13,16}$.

\section{Material y métodos}

Se llevó a cabo un estudio cuasi-experimental, donde el sujeto de estudio es su propio control. Los objetivos fueron: registrar la reducción de peso, IMC, exceso de peso corporal y porcentaje de exceso de peso de los pacientes con el uso de balón intragástrico ajustable a los 9 y 12 meses con respecto a su peso inicial, así como definir si existe diferencias estadísticamente significativas entre estas dos mediciones, así como contrastar el porcentaje de EPP a los 9 y 12 meses con las recomendaciones de la American Society for Gastrointestinal Endoscopy (ASGE) en 2015.

El registro de los pacientes a los cuales se les realizó colocación de balón intragástrico como tratamiento para la reducción de peso se llevó a cabo en el Servicio de Endoscopia gastrointestinal y cirugía endoscópica del Hospital General Dr. Fernando Quiroz Gutiérrez del Instituto de Seguridad y Servicios Sociales de los Trabajadores del Estado, durante el periodo comprendido del 1 de marzo de 2017 al 1 de marzo de 2018. Los pacientes fueron citados al Servicio de Endoscopia a los 9 y 12 meses para registro de pérdida de peso, se eligieron estos intervalos de seguimiento para documentar su impacto de la reducción de peso, ya que la mayoría de los dispositivos tienen una duración de 6 meses en promedio. El dispositivo utilizado fue el balón intragástrico ajustable Spatz $3^{\circledR}$, de la empresa Medical Great (Neck, NY), distribuido en México por Endomedics.

Los criterios de inclusión fueron: pacientes mayores de 18 años, con obesidad grado $1\left(\right.$ IMC $\left.>30 \mathrm{~kg} / \mathrm{m}^{2}\right) 0$ más, con valoración previa por el Servicio de Nutrición. Los criterios de eliminación fueron: pacientes que no acudan a sus citas de seguimiento con el Servicio de Nutrición, que no acudan al menos a un seguimiento endoscópico o a su retirada. Y el criterio de exclusión fue ser menor de 18 años.

Se requiere una valoración previa a la colocación del balón, las consultas de nutrición durante los primeros seis meses son mensuales y posteriormente cada dos meses hasta la retirada del balón, con una dieta restrictiva Gasto Energético Total (GET) menos 500 kcal, el porcentaje de apego reportado por el departamento de nutrición fue del $75 \%$ aproximadamente.

La colocación del balón intragástrico se realizó con un gastroscopio EG-760R ${ }^{\circledR}$, procesador Fujinon $\mathrm{BL} 7000^{\circledR}$, y un volumen inicial de aproximadamente $450-500 \mathrm{cc}$ en cada paciente, previa endoscopia diagnóstica.

Los datos se registraron en una hoja de cálculo (Excel ${ }^{\circledR}$, Microsoft), durante la consulta de seguimiento endoscópico a los 9 y a los 12 meses.

Al inicio, previo a la colocación del balón, se calculó el peso ideal para cada paciente por medio de la fórmula: Peso ideal $=I M C$ ideal $\times$ Talla al cuadrado. En el caso de los hombres el IMC ideal fue $23.5 \mathrm{y}$ en el caso de mujeres, 21.5.

Para el cálculo de exceso de peso corporal se utilizó la fórmula: Peso real - Peso ideal. Posteriormente durante los seguimientos se realizó el cálculo del porcentaje de EPP por medio de la siguiente fórmula: (Exceso de peso perdido $\times 100$ )/Exceso de peso.

Se empleó el software IBM SPSS ${ }^{\circledR}$ versión 24 para el análisis estadístico, obteniendo la frecuencia de presentación por sexo, la media de edad, peso, exceso de peso, IMC, EPP y PCTP, tanto inicial como a los 9 y 12 meses. Se aplicó la prueba de Shapiro-Wilk a cada variable cuantitativa continua para determinar su distribución, y se realizó comparación de medias con la prueba t de Student para determinar si existe diferencia estadísticamente significativa para el peso, el IMC y el exceso de peso.

Por último, contrastamos nuestros resultados con las recomendaciones emitidas por la ASGE publicadas en 2015. 


\section{Resultados}

Se evaluaron 15 pacientes, de los cuales cumplieron criterios de inclusión 11.

Para cada variable cuantitativa se aplicó la prueba de Shapiro-Wilk, que concluyó que seguían una distribución normal.

Se registraron las características de los 11 pacientes, resultando las medias como sigue: edad, 44.7 años (índice de confianza [IC] 95\%: 37.9-51.5); talla, $1.65 \mathrm{~m}$ (IC 95\%: 1.58-1.71); peso inicial, $127.5 \mathrm{~kg}$ (IC 95\%: 107.9-147.0); IMC, $46.77 \mathrm{~kg} / \mathrm{m}^{2}$ (IC 95\%: 39.83-53.70), y exceso de peso corporal, $66.99 \mathrm{~kg}$ (IC 95\%: 49.04-84.93). Se resumen las características generales en la tabla 1.

De los 11 pacientes que cumplieron los criterios, solo se presentaron 6 para su seguimiento a los 9 meses. A la revisión de 12 meses se presentaron los 5 pacientes que no acudieron a su primera revisión y solo uno del grupo previo.

Se obtuvieron los siguientes resultados:

- Grupo 9 meses de 6 pacientes. La media de peso alcanzada fue de $105.83 \mathrm{~kg}$ (IC 95\%: 76.46-135.20), para IMC fue de $41.06 \mathrm{~kg} / \mathrm{m}^{2}$ (IC 95\%: 29.43-52.70), el porcentaje de EPP fue del $34.50 \%$ (IC 95\%: 14.04-54.97), el porcentaje de PCTP fue del $17.20 \%$ (IC 95\%: 7.38-27.02), y para el peso perdido fue del $22.67 \mathrm{~kg}$ (IC 95\%: 5.97-39.36). Por último, la media del exceso de peso corporal fue de $49.70 \mathrm{~kg}$ (IC 95\%: 19.97-79.42) (Tabla 2).

- Grupo 12 meses de 6 pacientes. La media para el peso fue $104.83 \mathrm{~kg}$ (IC 95\%: 86.07-123.58); para IMC fue de $36.97 \mathrm{~kg} / \mathrm{m}^{2}$ (IC 95\%: 30.14-43.81); para el porcentaje de EPP de 40.52\% (IC 95\%: 22.57-58.47); el porcentaje de PCTP de $20.71 \%$ (IC 95\%: 10.68 30.75 ), y para el peso perdido de $29.15 \mathrm{~kg}$ (IC $95 \%$ : 9.78-48.51). El exceso de peso corporal en kilogramos promedio fue de 40.80 (IC 95\%: 24.09-57.50) (Tabla 3).

Además, se compararon las medias de las variables peso, IMC y exceso de peso corporal con la prueba $t$ de Student en cada grupo, con su media inicial y final correspondiente de los 9 y 12 meses. Se demostró una reducción estadísticamente significativa $(p<0.05)$ en el grupo de 12 meses.

Por último, el porcentaje de EPP recomendado por la ASGE en 2015 en relación a la terapia endoscópica para reducción de peso es de más del $25 \%$. En nuestra población se alcanzó un porcentaje de EPP del 34.50 y el $40.52 \%$ para 9 y 12 meses de tratamiento respectivamente (Tabla 4).
Tabla 1. Características generales de los pacientes con balón intragástrico Spatz $3^{\circledR}$

\begin{tabular}{|l|c|}
\hline Variables & Valores (IC 95\%) \\
\hline Número de pacientes & 11 \\
\hline Sexo femenino & 8 \\
\hline Sexo masculino & 3 \\
\hline Edad (años) & $44.7(37.9-51.5)$ \\
\hline Peso $(\mathrm{kg})$ & $127.5(107.9-147.0)$ \\
\hline Talla $(\mathrm{m})$ & $1.65(1.58-1.71)$ \\
\hline Exceso de peso $(\mathrm{kg})$ & $66.99(49.04-84.93)$ \\
\hline IMC $\left(\mathrm{kg} / \mathrm{m}^{2}\right)$ & $46.77(39.83-53.70)$ \\
\hline
\end{tabular}

IC: intervalo de confianza; IMC: índice de peso corporal.

Tabla 2. Grupo pacientes con balón intragástrico Spatz $3^{\circledR}$ durante 9 meses

\begin{tabular}{|l|c|c|c|}
\hline & Inicio & 9 meses & Valor de $\mathbf{p}$ \\
\hline Número de pacientes & 6 & 6 & \\
\hline Peso $(\mathrm{kg})$ & 128.50 & 105.83 & 0.10 \\
\hline Exceso de peso $(\mathrm{kg})$ & 72.36 & 49.70 & 0.10 \\
\hline IMC $\left(\mathrm{kg} / \mathrm{m}^{2}\right)$ & 49.77 & 41.06 & 0.11 \\
\hline Peso perdido $(\mathrm{kg})$ & - & 22.67 & - \\
\hline EPP $(\%)$ & - & 34.50 & - \\
\hline PPCT (\%) & - & 17.20 & - \\
\hline
\end{tabular}

IMC: índice de peso corporal; p: grado de significación; EPP: porcentaje de exceso de peso perdido; PPCT: porcentaje de peso corporal total perdido.

\section{Discusión}

Las opciones de tratamiento endoscópicas para reducción de peso son relativamente poco conocidas y en ocasiones se cuestiona su efectividad por la falta de evidencia en la población mexicana.

Presentamos la experiencia con el tratamiento endoscópico para reducción de peso tipo restrictivo. Contamos con resultados satisfactorios para la pérdida de peso alcanzada por los pacientes, basados en el porcentaje de EPP, siendo comparable con el alcanzado por otros autores (Machytka 2011, Brooks 2014), además de alcanzar las recomendaciones internacionales para dispositivos endoscópicos para reducción de peso (ASGE, 2015).

En nuestro reporte no se presentaron complicaciones graves ni muertes asociadas al balón intragástrico, 
Tabla 3. Grupo pacientes con balón intragástrico Spatz $3^{\circledR}$ durante 12 meses

\begin{tabular}{|l|c|c|c|}
\hline & Inicial & 12 meses & Valor de p \\
\hline Número de pacientes & 6 & 6 & \\
\hline Peso $(\mathrm{kg})$ & 133.98 & 104.83 & 0.012 \\
\hline Exceso de peso $(\mathrm{kg})$ & 69.95 & 40.80 & 0.006 \\
\hline IMC $\left(\mathrm{kg} / \mathrm{m}^{2}\right)$ & 47.17 & 36.97 & 0.012 \\
\hline Peso perdido $(\mathrm{kg})$ & - & 29.15 & - \\
\hline EPP $(\%)$ & - & 40.52 & - \\
\hline PPCT (\%) & - & 20.71 & - \\
\hline
\end{tabular}

IMC: índice de peso corporal; p: grado de significación; EPP: porcentaje de exceso de peso perdido; PPCT: porcentaje de peso corporal total perdido.

Tabla 4. Resultados de porcentaje de exceso de pérdida de peso alcanzado a los 9 y 12 meses y lo recomendado por la ASGE, 2015

\begin{tabular}{|l|c|c|c|}
\hline & 9 meses & 12 meses & ASGE, 2015 \\
\hline EPP $(\%)$ & 34.50 & 40.52 & $\geq 25$ \\
\hline IC $95 \%$ & $(14.0-54.9)$ & $(22.57-58.47)$ & \\
\hline
\end{tabular}

desafortunadamente, las complicaciones asociadas por el uso del balón intragástrico no fueron incluidas, por no ser objetivo del estudio. Sin embargo, no presentamos ninguna retirada del balón asociado por intolerancia, así como tampoco ningún ingreso a urgencias. Las molestias principales reportadas por los pacientes fueron náuseas, vómitos y dolor abdominal en las primeras semanas, de leve a moderada intensidad, las cuales cedieron con medicamentos orales.

La experiencia que aquí compartimos tiene limitaciones, principalmente en el diseño del estudio, el pequeño número de pacientes tratados y su seguimiento, así como por tratarse del primer reporte de la experiencia del balón intragástrico ajustable Spatz $3^{\circledR}$ en México. Esperamos que cada vez más existan más reportes de otros centros, y que nuestro trabajo sirva para motivar nuevos estudios y crezca el número de pacientes evaluados.

Por otro lado, estos son los resultados obtenidos por el uso del balón intragástrico para la reducción de peso, que no debemos olvidar que solo es parte del manejo multidisciplinario. Queda pendiente evaluar la eficacia en el mantenimiento del peso a lo largo del tiempo, siendo uno de los aspectos más relevantes, donde el manejo integral por nutrición y psicología juegan un papel fundamental para el éxito de cualquier tratamiento de reducción de peso.

\section{Conclusiones}

El uso del balón intragástrico ajustable como una alternativa en el tratamiento en la reducción de peso en la población mexicana ha mostrado, en nuestra experiencia, un éxito similar al reportado en la literatura y cumple con las recomendaciones internacionales. Cabe destacar que no deja de ser solo una parte del manejo multidisciplinario y que el mantenimiento del peso a largo plazo por parte de los pacientes aún está pendiente de demostrarse.

\section{Financiamiento}

La presente investigación no ha recibido ayudas específicas provenientes de agencias del sector público, sector comercial o entidades sin ánimo de lucro.

\section{Conflicto de intereses}

Los autores declaran no tener conflicto de intereses.

\section{Responsabilidades éticas}

Protección de personas y animales. Los autores declaran que para esta investigación no se han realizado experimentos en seres humanos ni en animales.

Confidencialidad de los datos. Los autores declaran que han seguido los protocolos de su centro de trabajo sobre la publicación de datos de pacientes.

Derecho a la privacidad y consentimiento informado. Los autores declaran que en este artículo no aparecen datos de pacientes.

\section{Bibliografía}

1. Li SH, Wang YJ, Zhang ST. Development of bariatric and metabolic endoscopy. Chin Med J (Engl). 2018;131(1):88-94.

2. Abu Dayyeh BK, Edmundowicz SA, Jonnalagadda S, Kumar N, Larsen M, Sullivan S, et al. Endoscopic bariatric therapies. Gastrointest Endosc. 2015;81(5):1073-86

3. Sullivan S, Kumar N, Edmundowicz SA, Abu Dayyeh BK, Jonnalagadda SS, Larsen M, et al. ASGE position statement on endoscopic bariatric therapies in clinical practice. Gastrointest Endosc. 2015;82(5):767-72.

4. Shamah-Levi T Cuevas-Nasu L Dommarco-Rivera J Hernandez-Avila M. Encuesta Nacional de Salud y Nutrición de Medio Camino 2016. (ENSANUT MC 2016). México: Instituto Nacional Salud Pública; 2016. Disponible en: http://promocion.salud.gob.mx/dgps/descargas1/doctos_2016/ ensanut mc 2016-310oct.pdf

5. Biener A, Cawley J, Meyerhoefer C. The medical care costs of youth obesity: An instrumental variables approach [Internet]. Cambridge, MA: National Bureau of Economic Research, NBER Working Paper No. 23682; 2017. Disponible en: http://www.nber.org/papers/w23682.pdf

6. Barquera S, Nonato IC, Barrera LH, Rivera J. Evidencia para la política pública en salud. Obesidad en adultos: los retos de la cuesta abajo. ENSANUT Encuesta Nac Salud y Nutr. 2012;2012:1-5. 
7. Rivera J, Hernández M, Aguilar C, Vadillo F. Obesidad en México: Recomendaciones para una Política de Estado. Unam. 2013;53(9).

8. Bray GA, Frühbeck G, Ryan DH, Wilding JPH. Management of obesity. Lancet. 2016:387(10031):1947-56.

9. Prevención, diagnóstico y tratamiento del sobrepeso y la obesidad exógena [Internet]. México: CENETEC, Guías de Práctica Clínica, actualización 2012; 2012. Disponible en: www.cenetec.salud.gob.mx/interior/gpc.htm

10. Chang SH, Stoll CRT, Song J, Varela JE, Eagon CJ, Colditz GA. The effectiveness and risks of bariatric surgery an updated systematic review and meta-analysis, 2003-2012. JAMA Surg. 2014;149(3):275-87.

11. Angrisani L, Santonicola A, lovino P, Formisano G, Buchwald H, Scopinaro N. Bariatric surgery worldwide 2013. Obes Surg. 2015;25(10):1822-32.

12. Vargas EJ, Abu Dayyeh BK. Endoluminal bariatric and metabolic interventions. Tech Gastrointest Endosc. 2015;17(4):171-7.

13. Espinet-Coll E, Nebreda-Durán J, Gómez-Valero JA, Muñoz-Navas M, Pujol-Gebelli J, Vila-Lolo C, et al. Current endoscopic techniques in the treatment of obesity. Rev Esp Enfermedades Dig. 2012;104(2):72-87.
14. Brooks J, Srivastava ED, Mathus-Vliegen EMH. One-year adjustable intragastric balloons: Results in 73 consecutive patients in the UK. Obes Surg. 2014;24(5):813-9.

15. Abu Dayyeh BK, Edmundowicz S, Thompson CC. Clinical practice update: Expert review on endoscopic bariatric therapies. Gastroenterology. 2017;152(4):716-29.

16. Genco A, Dellepiane D, Baglio G, Cappelletti F, Frangella F, Maselli R, et al. Adjustable intragastric balloon vs non-adjustable intragastric baIloon: Case-control study on complications, tolerance, and efficacy. Obes Surg. 2013;23(7):953-8.

17. Russo T, Aprea G, Formisano C, Ruggiero S, Quarto G, Serra R, et al. BioEnterics Intragastric Balloon (BIB) versus Spatz Adjustable Balloon System (ABS): Our experience in the elderly. Int J Surg. 2017:38:138-40.

18. Machytka E, Klvana P, Kornbluth A, Peikin S, Mathus-Vliegen LEM, Gostout C, et al. Adjustable intragastric balloons: A 12-month pilot trial in endoscopic weight loss management. Obes Surg. 2011; 21(10):1499-507. 